\section{Magnetic Resonance Imaging in Movement Disorders: A Guide for Clinicians and Scientists}

\author{
P. Tuite and A. Dagher, Eds.
}

New York, NY: Cambridge University Press, 2013, 291 pages, $\$ 150$

Magnetic Resonance Imaging in Movement Disorders is a useful guide for general readers, including nuclear medicine physicians, who may not be experts in the various MR imaging technologies. This well-organized book provides an opportunity to become current on recent progress in these technologies and to learn of the future prospects for routine use of MR imaging in the characterization and treatment of movement disorders.

Movement disorders, especially Parkinson disease, have long been treated by medication, but medication rarely modifies their clinical course. Although treatment may initially look successful, the disease may afterward become intractable, with various complications. This book helps readers appreciate how difficult is the disease-modifying treatment of movement disorders. In Parkinson disease, although MR imaging has helped us to understand the nature of the disease, reversal of its neurodegeneration and associated abnormalities is still in vain. Other movement disorders are beyond our comprehension; elucidating their pathophysiologic basis using MR imaging is still in its infancy.

The first half of this book is devoted to Parkinson disease and related syndromes; the second half to other movement disorders. Readers get a glimpse of current progress in examining the nature of these diseases using MR imaging and how their elucidation can affect disease-modifying "definitive treatment" such as deep-brain stimulation. The book describes how the development of not only MR instrumentation but also MR imaging techniques-as well as PET and SPECT - has contributed to the evaluation of Parkinson disease and other movement disorders. MR imaging, either standard Tesla or high-field, has enabled morphometric analysis, iron imaging using $\mathrm{T} 2 *$ signal, magnetization transfer imaging, susceptibilityweighted imaging, resting/activation functional MR imaging, MR spectroscopy, and diffusion tensor imaging.

The section on Parkinson disease presents neuroimaging studies on cognitive impairment and the related structural changes in gray and white matter, starting with the iron-imaging technique using $\mathrm{T} 2 *$ mapping and susceptibility-weighted imaging for iron quantification and differential diagnosis of parkinsonian syndromes. The use of functional MR imaging to study the various clinical aspects such as motor, cognition, and mood abnormalities is presented and the literature summarized. The association between resting-state functional MR imaging findings and clinical findings in Parkinson disease is then explained. Planning of surgical deep-brain stimulation-the most useful clinical application of MR imaging in
Parkinson disease-follows. Readers will discover how MR sequences such as susceptibility-weighted imaging and $\mathrm{T} 2 *$ and high-magneticfield MR imaging enable characterization of such small targets as the subthalamic nucleus and the globus pallidus internus, as well as how the success of deep-brain stimulation depends heavily on MR imaging. Also detailed is the application of MR spectroscopy to movement disorders - a technique that, despite its potential to clarify pathophysiology, is not definitive enough for routine clinical application.

The section on movement disorders other than Parkinson disease is concise and interesting, summarizing the utility of MR imaging in Huntington disease, Gilles de la Tourette syndrome, dystonia, multiple-system atrophy, ataxia, and tauopathy. Movement disorders are sometimes made more clinically challenging by being associated with dementia; the tauopathy chapter elucidates the potential for MR imaging to help us understand and characterize these complex syndromes. Unbiased, balanced descriptions of the utility of MR imaging are provided. Multiple representative cases of normal and abnormal MR findings in various movement disorders are presented. These cases enlighten readers to the fact that routine MR imaging findings in these movement disorders, although seeming ambiguous or nonspecific, may prove more informative than meets the eye if better methods of imaging and analysis are devised.

Because the basics of MR imaging physics are covered only briefly, the book might prove challenging for those unfamiliar with MR techniques. However, this shortcoming is balanced by the structured, in-depth descriptions and well-organized chapters, as well as by the thorough explanations of the various analysis methods: voxel-based morphometry for structural MR imaging, resting-state functional MR imaging, motor activation functional MR imaging, MR spectroscopy, and network analysis based on the statistical parametric mapping method. This information helps the reader understand the promise of MR imaging for movement disorders despite its present limitations even with the best analytic approaches. This book should appeal to any nuclear medicine physician, neurologist, or radiologist who is interested in understanding the application of MR imaging and related methods to movement disorders.

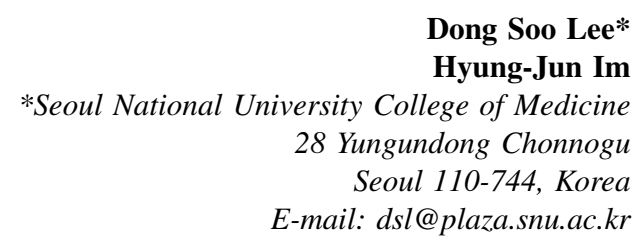

Published online Apr. 9, 2015.

DOI: $10.2967 /$ jnumed.115.157461

COPYRIGHT (C) 2015 by the Society of Nuclear Medicine and Molecular Imaging, Inc. 